\title{
WRN participates in translesion synthesis pathway through interaction with NBS1.
}

\author{
AUTHOR(S): \\ Kobayashi, Junya; Okui, Michiyo; Asaithamby, \\ Aroumougame; Burma, Sandeep; Chen, Benjamin P C; \\ Tanimoto, Keiji; Matsuura, Shinya; Komatsu, Kenshi; Chen, \\ David J
}

\section{CITATION:}

Kobayashi, Junya ... [et al]. WRN participates in translesion synthesis pathway through interaction with NBS1.. Mechanisms of ageing and development 2010, 131(6): 436-444

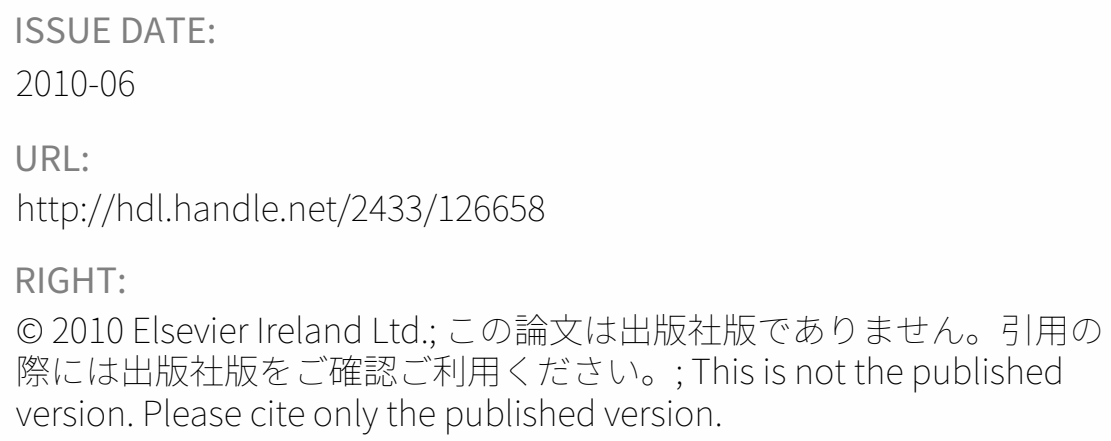




\section{WRN participates in translesion synthesis pathway through interaction with NBS1.}

Junya Kobayashi ${ }^{1,6}$, Michiyo Okui ${ }^{2}$, Aroumougame Asaithamby ${ }^{3}$, Sandeep Burma ${ }^{3}$, Benjamin PC Chen ${ }^{3}$, Keiji Tanimoto ${ }^{4}$, Shinya Matsuura ${ }^{5}$, Kenshi Komatsu ${ }^{1}$, David J Chen $^{3,6}$.

1 Deapartment of Genome Repair Dynamics, Radiation Biology Center, Kyoto University, Kyoto 606-8501, Japan

${ }^{2}$ Biomedical Engineering Center, Toin University of Yokohama, Yokohama 225-8502, Japan

${ }^{3}$ Division of Molecular Radiation Biology, Department of Radiation Oncology, University of Texas Southwestern Medical Center at Dallas, Dallas, TX 75390-9187, USA

${ }^{4}$ Department of Oral and Maxillofacial Radiology, Graduate School of Biomedical Sciences, Hiroshima University, Hiroshima 734-8553, Japan

${ }^{5}$ Department of Radiation Biology, Research Institute for Radiation Biology and Medicine, Hiroshima University, Hiroshima 734-8553, Japan

${ }^{6}$ Correspondences should be address by Dr. Junya Kobayashi

E-mail: jkobayashi@house.rbc.kyoto-u.ac.jp

Phone: +81-75-753-7554; Fax: +81-75-753-7564

${ }^{6}$ Correspondences should be address by Dr. David J Chen

E-mail: David.Chen@UTSouthwestern.edu

Phone: +1-214-648-5597; Fax: +1-214-648-5595

Number of figures; 5

Number of page; 31

Running title: WRN participates in translesion synthesis

Keywords: WRN, NBS1, TLS, PCNA, Rad18 


\section{Abstract}

Werner syndrome (WS), caused by mutation of the $W R N$ gene, is an autosomal recessive disorder associated with premature aging and predisposition to cancer. WRN belongs to the RecQ DNA helicase family, members of which play a role in maintaining genomic stability. Here, we demonstrate that WRN rapidly forms discrete nuclear foci in an NBS1-dependent manner following DNA damage. NBS1 physically interacts with WRN through its FHA domain, which interaction is important for the phosphorylation of WRN. WRN subsequently forms DNA damage-dependent foci during the $\mathrm{S}$ phase, but not in the G1 phase. WS cells exhibit an increase in spontaneous focus formation of pol $\eta$ and Rad18, which are important for translesion synthesis (TLS). WRN also interacts with PCNA in the absence of DNA damage, but DNA damage induces the dissociation of PCNA from WRN, leading to the ubiquitination of PCNA, which is essential for TLS. This dissociation correlates with ATM/NBS1-dependent degradation of WRN. Moreover, WS cells show constitutive ubiquitination of PCNA and interaction between PCNA and Rad18 E3 ligase in the absence of DNA damage. Taken together, these results indicate that WRN participates in the TLS pathway to prevent genomic instability in an ATM/NBS1-dependent manner. 


\section{Introduction}

DNA double-strand breaks (DSBs) are often generated by exposure to ionizing radiation (IR) and DNA-damaging agents, or the stall/collapse of DNA replication forks. Unrepaired DSBs cause genomic instability and promote tumorigenesis; therefore, upon DSB induction, cells promptly activate cell cycle checkpoints and DNA repair mechanisms. Cells derived from individuals with DNA damage-sensitive genetic disorders have been extensively used to study the DNA damage response (DDR). WS is also categorized as a DNA damage-sensitive disease (Cheng et al., 2007; Gray et al., 1997). Individuals with WS exhibit symptoms of premature aging, including graying and loss of hair, wrinkling and ulceration of skin, atherosclerosis, osteoporosis, cataracts, and an elevated incidence of unusual types of cancer. Primary cells from WS patients exhibit premature replicative senescence and genomic instability such as chromosomal rearrangements and genomic deletions. WS cells also show hypersensitivity to several types of DNA damaging agents including 4-nitroquinoline-1-oxide (4NQO), cross-linking agents (such as mitomycin $\mathrm{C}$ and cisplatin), camptothecin (CPT), and hydroxyurea (Poot et al., 1999; Poot et al., 2002; Constantinou et al., 2000). These reports suggest that the gene responsible for WS plays an important role in one or more genome maintenance pathways.

Mutation of the $W R N$ gene, a member of the RecQ DNA helicase family, results in WS (Yu et al., 1997; Gray et al., 1997). Mutations in other family members, such as BLM and RECQ4, are responsible for causing two other cancer-prone and premature aging syndromes, Bloom and Rothmund-Thomson, respectively. Consistent 
with other RecQ helicases, WRN protein possesses 3' to 5' DNA helicase activity; however, it is the only human RecQ member that also has 3' to 5' exonuclease activity. Although the identity of the physiological substrate(s) of WRN is not yet clear, several evidences indicate that WRN preferentially acts on replication or DNA repair mechanisms. WRN protein localizes to the nucleoli in the absence of DNA damage, but migrates from nucleoli to discrete nuclear foci after exposure to several DNA-damaging agents such as 4NQO, etoposide, hydroxyurea (HU), or CPT (Sakamoto et al., 2001; Cheng et al., 2004; Cheng et al., 2005). Further, WRN interacts physically and functionally with a number of DNA metabolic pathway proteins, such as replication protein A (RPA), proliferating cell nuclear antigen (PCNA), polymerase $\delta$, flap endonuclease 1 (FEN-1), RAD51, RAD52, the hMRE11/hRAD50/NBS1 (MRN) complex, and $\mathrm{Ku}$ heterodimer (Sakamoto et al., 2001; Cheng et al., 2005; Brosh et al., 2001; Yannone et al., 2001; von Kobbe et al., 2002; Otterlei et al., 2006). These reports suggest that WRN could play an important role in the DNA damage response.

Mutation of NBS1 causes Nijmegen breakage syndrome (NBS). NBS patient cells show radio-resistant DNA synthesis, radiation hyper-sensitivity, and genomic instability (Kobayashi et al., 2004). NBS1 protein forms a complex with hMRE11 nuclease and hRAD50, and this MRN complex functions in DNA DSB repair by homologous recombination (HR). Therefore, the genomic instability in NBS patients could be due to a defect in HR (Tauchi et al., 2002). The function of NBS1 in HR requires IR-induced focus formation of the MRN complex (Sakamoto et al., 2007), and we previously reported that focus formation by NBS1 is dependent on direct interaction 
of NBS1 with $\gamma-\mathrm{H} 2 \mathrm{AX}$ at DNA damage sites (Kobayashi et al., 2002). Current reports also suggest a novel role of NBS1 in alternative NHEJ pathway (Rass et al., 2009; Xie et al., 2009). NBS1 also interacts with ATM through the C-terminal region and this interaction is indispensable for the recruitment of ATM to DSB sites and the phosphorylation and activation of cell cycle checkpoints by ATM (Falck et al., 2005; Iijima et al., 2008). Thus, the NBS1 complex functions in the regulation of both DSB repair and cell cycle checkpoints following the generation of DSBs.

DNA damage induced by UV, mitomycin $\mathrm{C}$ (MMC), and Methyl methane-Sulfonate cause stalling or collapse of DNA replication forks. Such replication blocks are circumvented by TLS (translesion synthesis) DNA polymerases (Lehmann, 2006). TLS DNA polymerases are specialized DNA polymerases whose primary function is to insert nucleotides across DNA lesions at replication-blocked sites. Eukaryotes are endowed with several TLS DNA polymerases, each presumably responsible for the bypass of a specific lesion or class of lesions. Human cells have four

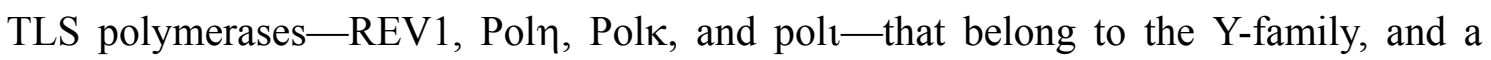
B-family polymerase, pol $\zeta$. Of these, Pol $\eta$ is implicated in error-free bypass of UV-induced cyclobutane pyrimidine dimers (CPD) based on in vivo and in vitro evidence (Masutani et al., 2000). In UV-irradiated human cells, Pol $\eta$ is recruited to DNA damage sites, presumably at sites with stalled replication forks, and interacts with ubiquitinated PCNA (Watanabe et al., 2004; Tissier et al., 2004; Stelter and Ulrich 2003). Moreover, the ubiquitination of PCNA is carried out in a RAD6/RAD18-dependent manner (Hoege et al., 2002). Further, TLS DNA polymerases 
including Pol $\eta$, can be error-prone across normal bases (Casali et al., 2006). Hence, the regulation of PCNA ubiquitination is very important to suppress the replication errors caused by TLS DNA polymerase and to maintain genomic stability.

In this paper, we investigated the role of WRN in several DNA damage responses. WRN showed DNA damage-dependent relocation from the nucleolus to the nucleoplasm and discrete nuclear foci only during the $\mathrm{S}$ phase. This focus formation occurred in an NBS1-dependent manner. NBS1 interacts with WRN through the FHA domain, and this interaction contributes to the ATM-dependent phosphorylation of WRN. Further, WRN interacts with PCNA, but gets dissociated in response to DNA damage. WRN cells showed constitutive ubiquitination of PCNA and interaction of PCNA with Rad18, suggesting that WRN might suppress PCNA ubiquitination through this interaction. Moreover, WRN protein was degraded in response to DNA damage, but AT and NBS cells did not show this degradation. Taken together, our results indicate that ATM/NBS1-dependent phosphorylation of WRN is important for WRN degradation and might abolish the suppressive effect of WRN on TLS.

\section{Material and Methods}

\subsection{Cell lines}

Werner fibroblast cell line (73-26 cells) and WRN-complemented cells were used as described previously (Perry et al., 2006). NBS fibroblast cell line, GM07166VA7, was established by SV40-transformation of GM07166 cells (Tauchi et al., 2001), which 
were provided from NIGMS Cell Repository. We also used SV40-transformed MRC5SV fibroblasts, and AT5BIVA fibroblasts, which was provided from NIGMS Cell Repository (Kobayashi et al., 2002). Normal lymphoblastoid cell line, GM2184 and AT lymphoblastoid cell line, CSA were also obtained from NIGMS Cell Repository. NBS lymphoblastoid cell line, 94p548, was kindly supplied by Dr. K.Sperling. Human primary fibroblast, TIG-3 cells was supplied by Dr. Y. Mitsui and their doubling time is 24 hours. hTERT-introduced human primary fibroblast, 48BR was supplied by Dr. P. Jeggo. GFP-pol $\eta$-expressing HeLa cells were obtained from Dr. F. Hanaoka (Sekimoto et al., 2010). All fibroblast cells were cultured in DMEM (Sigma) supplemented with 10\% FBS (Invitrogen) and antibiotics, and lymphoblastoid cells were cultured in RPMI (Sigma) supplemented with 10\% FBS (Invitrogen) and antibiotics.

\subsection{Western blot analysis}

Western blot analysis was performed as previously reported (Kobayashi et al, 2002). To detect target proteins, we used the following primary antibodies: anti-WRN (Santa Cruz Biotechnology: sc-5629), anti-NBS1 (Tauchi et al., 2001), anti-phospho-SMC1[pS966] (Bethyl Laboratories: A300-050A), anti-phospho Chk2 [pT68] (Cell Signaling Technology: \#2661), anti-Chk2 (Cell Signaling Technology: \#2662), anti-Mre11 (Novus Biologicals: NB100-142), anti-PCNA (Santa Cruz Biotechnology: sc-56), anti-gammaH2AX (Upstate: \#05-636), anti-phospho ATM [pA1981] (Rockland Inc: 600-401-400) anti-Rad18 (Abnova Corporation: 
H00056852-M01) antibodies. Primary antibodies were detected with HRP-conjugated anti-rabbit IgG or anti-mouse IgG antibodies (GE Helthcare), and then visualized with an ECL plus chemiluminescence system (GE Helthcare).

\subsection{Immunoprecipitation analysis}

Immunoprecipitation was performed as previously reported (Kobayashi et al., 2002). Briefly, DNA damage-treated or untreated cells were lysed in IP buffer (150 mM sodium chloride, $10 \mathrm{mM}$ HEPES at $\mathrm{pH} 7.4$ and $0.5 \%$ NP40) containing a protease inhibitor cocktail (Roch) and sodium orthovanadate for 15 min. Lysates were centrifuged at $20,000 \times \mathrm{g}$ for $30 \mathrm{~min}$ to remove un-solubulized debris. Lysates were pre-cleared with protein A-Sepharose beads (GE healthcare), and immunoprecipitation was performed by incubating the samples with anti-NBS1 polyclonal antibodies, anti-WRN polyclonal antibodies or anti-Rad18 polyclonal antibodies at $4 \circ \mathrm{C}$ for 1 hour. Immuno-complexes were precipitated with protein A-Sepharose beads and washed with IP buffer and detected by Western blot.

\subsection{Immunofluorescent staining}

Immunostaining was performed as previously reported (Kobayashi et al., 2002). Cells grown on a glass slide were fixed with cold methanol for $15 \mathrm{~min}$, rinsed with cold acetone for several times, and then air-dried. To detect chromatin-associated WRN foci, cells were treated with $0.5 \%$ Triton X-100/PBS for $5 \mathrm{~min}$ before methanol fixation. Alexa-488-conjugated anti-rabbit $\operatorname{IgG}$ or Alexa-546-conjugated anti-mouse IgG 
antibodies (Molecular Probes) were used for visualization of foci with anti- $\gamma-\mathrm{H} 2 \mathrm{AX}$ antibody (Upstate: \#05-636), anti-NBS1 antibody (Tauchi et al., 2001), anti-phospho-DNA-PKcs (T2609) antibody (Chan et al., 2002), anti-MDC1 antibody (Bethyl Laboratories: A300-051A), anti-WRN antibody (Santa Cruz Biotechnology: sc-5629) or anti-Rad18 (Abnova Corporation: H00056852-M01) antibody.

\subsection{SupF mutation assay}

SupF assay was performed as previously reported (Parris and Seidman, 1992; Sekimoto et al., 2010). The pSP189 plasmid DNA $(50 \mu \mathrm{g})$ dissolved in $1 \mathrm{ml}$ of TE buffer $(10 \mathrm{mM}$ Tris [pH 7.5], $1 \mathrm{mM}$ EDTA) was irradiated with $200 \mathrm{~J} / \mathrm{m}^{2} \mathrm{UV}$ in a sterile plastic $60 \mathrm{~mm}$ tissue culture dish. The plasmid DNA was then precipitated with ice-cold ethanol, redissolved in TE buffer, and transfected into the indicated cells using Lipofectamine 2000 (Invitrogen). After $48 \mathrm{hr}$, the plasmid DNA was isolated, and mutation frequency of $\sup F$ was determined as described (Choi and Pfeifer, 2005).

\section{Results}

\section{WRN is relocated to the nucleoplasm in an NBS1-dependent manner}

WS cells show sensitivity to several types of DNA damage induced by 4NQO, UV, CPT, MMC, and etoposide, suggesting that the product of the WRN gene might play an important role in the DNA damage response. As several DDR factors are known to be recruited to DNA damage sites and form discrete nuclear foci, we investigated the 
relocation of WRN following several types of DNA damage (Fig 1A). WRN is confined to the nucleoli without DNA damage, but WRN relocated from nucleoli to the nucleoplasm following treatment with $\gamma$-ray, bleomycin, hydroxyurea (HU), CPT, and etoposide, and formed discrete minute foci. $\gamma$-ray, bleomycin, and etoposide induce DNA DSBs directly, but HU and CPT lead to different types of DNA damage, suggesting that WRN may respond to several types of DNA damage. Thus far, relocation and focus formation of WRN are suggested to be the late events ( $4 \mathrm{~h}$ or later after DNA damage) in DDR; however, we observed these events at 1 hour after treatment with $\gamma$-ray and CPT treatment (Fig. 1B and Supplementary Fig. 1). Cheng et al. reported that late focus formation of WRN is dependent on NBS1 following irradiation (Cheng et al., 2004). We investigated whether these early response events of WRN requires NBS1. NBS1-defective NBS cells did not exhibit formation of chromatin-associated WRN foci following the treatment with $\gamma$-ray (Fig. 1B). Although approximately $80 \%$ of normal cells showed WRN focus formation following exposure to $\gamma$-rays, WRN focus formation was seen in less than $5 \%$ of NBS cells (Supplementary Fig. 2). NBS cells also showed a defect in WRN focus formation after CPT treatment (Supplementary Fig.1). Furthermore, WRN interacted with NBS1 without and with irradiation (Fig. 1C and Supplementary Fig. 3). These results suggest that the interaction of WRN with NBS1 is important for the early focus formation of WRN in response to DNA damage. Moreover, WRN-defective WS cells formed IR-induced foci of NBS1, $\gamma$-H2AX, MDC1, and phospho-DNA-PKcs, which responses occur at early times after irradiation (Fig. 1D). Collectively, our results indicate that the 
relocation and focus formation of WRN might be a secondary or later event that occurs in an NBS1-dependent manner.

\section{N-terminus of NBS1 is required for the interaction with WRN and DNA damage-dependent phosphorylation of WRN}

WRN has been reported to be phosphorylated by ATM/ATR in response to DNA damage (Pichierri et al., 2003). NBS1 is a regulator of ATM kinase in the DSB-induced response; hence, we speculated that the NBS1-dependent focus formation of WRN is essential for its phosphorylation in response to DNA damage. As WRN phosphorylation leads to reduced mobility of WRN bands in western blot analysis, we tested for WRN phosphorylation in NBS cells by Western blot analysis (Fig. 2A). Wild type cells showed reduced mobility of WRN bands at 1 and 4 hours after exposure to 5 Gy of $\gamma$-ray indicating phosphorylation of WRN. However, WRN was not phosphorylated in NBS and AT cells. Further, anti-pS/pTQ antibody, which can detect ATM/ATR dependent phosphorylations by Western blot or immunoprecipitation analysis, immunoprecipitated WRN protein as well as SMC1, which is known to contain S/TQ motifs phosphorylated by ATM (Supplementary Fig. 4A), and the ATM inhibitor KU55933 repressed DNA damage-induced phosphorylation of WRN (Supplementary Fig. 4B), suggesting that WRN could be phosphorylated in an ATM/NBS1-dependent manner. As the S/T-Q motif can also be phosphorylated by DNA-PK, we also examined the contribution of this kinase. Treatment with a DNA-PK inhibitor (NU7026) did not influence the band mobility (phosphorylation) of 
WRN following IR, although non-irradiated cells also showed WRN phosphorylation (Supplementary Fig. 5). Thus, DNA-PK may not contribute to IR-induced WRN phosphorylation.

To dissect the WRN-NBS1 interaction further, we investigated the interaction of endogenous WRN with exogenous mutated NBS1 by immunoprecipitation. Wild-type NBS1 (MRC and Full) was co-immunoprecipitated with WRN by anti-NBS1 antibody, but NBS1 with mutation in the FHA domain did not associate with WRN (Fig. 2C). NBS1, with deletion of both MRE11-binding and ATM-interaction motifs (R2), interacted with WRN. Further, NBS1, mutated at the BRCT domain, also interacted with WRN. Moreover GST-tagged N-terminal NBS1 fragment could co-immunoprecipitate endogenous WRN, but C-terminal NBS1 fragment could not (Supplementary Fig. 6). Collectively, our results indicated that the FHA domain of NBS1 might be important for the interaction with WRN. Furthermore, NBS cells expressing NBS1, mutated in the FHA domain, did not show IR-dependent phosphorylation of WRN (Fig. 2D), suggesting that the interaction of WRN with NBS1 through its FHA domain contributes to WRN phosphorylation. As both phosphorylation and focus formation of WRN are induced by DNA damage, we investigated whether the phosphorylation of WRN contributes to its focus formation. However, ATM-deficient AT cells showed WRN focus formation, and caffeine, an inhibitor of ATM and ATR did not abolish WRN focus formation (Fig. 2E). Taken together, these results indicate WRN focus formation might not require its phosphorylation and WRN may be phosphorylated after recruitment (focus formation) to the DNA damage site. 


\section{WRN functions in the $\mathrm{S}$ phase-dependent DNA damage response.}

WS cells show increased sensitivity to several DNA damaging agents such as 4NQO, UV, CPT, MMC, and etoposide. These agents induce different types of DNA damage that can stall DNA replication forks and this stalling could cause $\gamma$-H2AX formation in an ATR-dependent manner (Stiff et al., 2005). Spontaneous DNA damage could also stall DNA replication and induce such $\gamma-\mathrm{H} 2 \mathrm{AX}$ formation (Bartkova et al., 2005). WS cells showed an increase in $\gamma-\mathrm{H} 2 \mathrm{AX}$ formation without irradiation as compared to WRN-complemented cells (Fig. 3A). WRN-knockdown cells also show an increase in $\gamma$-H2AX without and with DNA damage, while WRN-knockdown did not influence the expression of H2AX (Supplementary Fig. 10). These results suggest that WS cells may be unable to repair replication-coupled DNA damage. Moreover, WRN focus formation after etoposide treatment was detected only in the S phase, but this was not observed in G1 phase cells, although phophorylated DNA-PKcs foci were observed in G1 phase cells only (Fig. 3B; Chen et al., 2005). We also confirmed that etoposide treatment could induce $\gamma$-H2AX foci in both G0/G1 phase (PCNA-negative: $89.4 \%$ ) and S phase (PCNA-positive: 64.9\%) cells (Supplementary Fig. 7). Collectively, these results indicate that WRN may function in DNA replication ( $\mathrm{S}$ phase)-dependent damage response. Recently, it has been known that TLS is important to maintain genomic integrity to circumvent $S$ phase-dependent damage. As poln, one of the TLS polymerases, forms nuclear foci at S phase-dependent DNA damage sites (Watanabe et al, 2004; Sekimoto et al., 2010), we investigated the focus formation of pol $\eta$, with or 
without UV irradiation (Fig. 3C and Supplementary Fig. 8A). In agreement with the results of Fig. 3A, WRN-knockdown cells showed an increase in poln focus formation with or without DNA damage. Focus formation of Rad18, which is important for activation of TLS pathway (Watanabe et al, 2004), also increased in WS cells compared to normal cells (Fig. 3D and Supplementary Fig. 8B). Although we cannot completely rule-out the possibility that increased focus formation in WS cells reflects inhibition, rather than activation, of TLS pathways, WS cells increased polymerase $\eta$-related mutation rates (Fig. 3E). We also confirmed that these mutations did not contain large deletion by sequence analysis (data not shown). These results suggest that WRN may function in the S phase-dependent DNA damage response, particularly in a fashion that restrains the TLS pathway.

\section{WRN might mediate translesion DNA synthesis via its interaction with PCNA}

As TLS is known to be activated by mono-ubiquitination of PCNA by Rad18 (Lehmann 2006), the results of Fig. 3CDE suggest that WRN has a functional interaction with PCNA. Upon immunoprecipitation with anti-WRN antibody without CPT treatment, PCNA was co-precipitated (Fig. 4A). But this interaction disappeared after CPT treatment, although CPT could induce DNA replication-dependent damage including DSBs through the generation of SSBs. However, this interaction increased in NBS cells without damage, and the dissociation of PCNA from WRN decreased [the decrease ratio for interaction; normal: 0.49 and NBS: 0.71] (Fig. 4B), while the expression of PCNA 
was unchanged after DNA damage in both normal and NBS cells (Supplementary Fig. 9AB). Immunoprecipitation with an anti-PCNA antibody also showed similar results in NBS cells at 8 hours after CPT treatment (Supplementary Fig. 9C). hMRE11-deficient AT-LD cells, which also exhibit remarkable decreases in NBS1 expression, showed no dissociation of PCNA from WRN after CPT treatment (Supplementary Fig. 9D). Collectively, NBS1 might be important for this dissociation. Furthermore, we examined the interaction between PCNA and Rad18 by co-immunoprecipitation (Fig. 4C). Rad18 did not interact with PCNA in normal cells without DNA damage, but after CPT treatment, interaction with PCNA was observed. In agreement with this, PCNA was mono-ubiquitinated in normal cells after CPT treatment (Fig. 4D). Surprisingly, WS cells exhibited association of Rad18 with PCNA and PCNA mono-ubiquitination with and without CPT treatment (Fig. 4C). Additionally, WRN-knockdown cells also showed mono-ubiquitination of PCNA at higher levels with and without CPT treatment (Supplementary Fig. 10). Moreover, decreases in total WRN at both 1 and 4 hours after 10 Gy of $\gamma$-irradiation were observed in normal lymphoblastoid cells (Supplementary Fig 11A). These results suggest that the interaction of WRN with PCNA may regulate the interaction between PCNA and Rad18 and its mono-ubiquitination, and TLS. However, WRN did not show any direct physical interaction with Rad18 (Supplementary Fig. 9A), suggesting that WRN might not regulate the activity of Rad18 directly. Further, Fig. 4D shows that the amount of WRN decreased after DNA damage probably because of degradation. Protease inhibitor MG132 treatment attenuated this degradation following IR (Supplementary Fig. 11B). 
However, in both AT and NBS cells, which did not exhibit DNA damage-dependent phosphorylation of WRN (Fig. 2A), also did not show WRN degradation after DNA damage (Fig. 4E). Furthermore, treatment with the ATM inhibitor (KU-55933) abolished this degradation (Fig. 4F) and NBS1-knockdown cells exhibited similar results (supplementary Fig. 12A). Moreover, these NBS and AT cells did not show clear ubiquitination of PCNA after CPT treatment (Supplementary Fig. 12B). As NBS cells are defective in DSB repair and could accumulate spontaneous DNA damage, spontaneous ubquitination of PCNA in WS cells might not be due to an accumulation of spontaneous DNA damage. These results suggest that ATM/NBS1-dependent WRN phosphorylation may be important for its degradation. Taken together, these data indicated that WRN might participate in TLS pathway through its interaction with PCNA and its degradation.

\section{Discussion}

Here, we show that WRN responds to S phase-dependent DNA damage and forms discrete nuclear foci. This focus formation is dependent on interaction with NBS1, and is important for the phosphorylation of WRN. Furthermore, this ATM/NBS1-dependent WRN phosphorylation might lead to WRN degradation in response to DNA damage. WRN forms DNA damage-dependent foci only during $\mathrm{S}$ phase and WS cells showed increases in poln and Rad18 focus formation. Furthermore, PCNA dissociates from the WRN complex after DNA damage in an NBS1-dependent 
manner. Moreover, WS cells showed an increase in the interaction between PCNA and Rad18 and spontaneous mono-ubiquitination of PCNA. Taken together, our results indicate that WRN could participate in the repressive regulation to PCNA/Rad18-dependent TLS through its interaction with PCNA and its phosphorylation/degradation in an S phase-dependent DNA damage pathway (Fig. 5).

WRN protein possesses several SQ/TQ motifs that can be phosphorylated by ATM, DNA-PK, or ATR in response to DNA damage (Matsuoka et al., 2007; Pichierri et al., 2003). We also showed that WRN was phosphorylated at SQ/TQ motif site(s) in an ATM/NBS1-dependent manner (Fig. 2A and Supplementary Fig. 4AB), which is corroborated by Pichierri's report showing that WRN was phosphorylated in response to DNA-replication-related damage by CPT, HU, or UV and that this phosphorylation was attenuated in ATM or ATR-deficient cells (Pichierri et al., 2003). Furthermore, we found that wild type cells showed a decrease in WRN protein following DNA damage, but neither AT nor NBS cells did (Fig. 4DEF and Supplementary Fig. 12A). Furthermore, the protease inhibitor MG132 attenuated this decrease (Supplementary Fig. 11B), suggesting that DNA damage-dependent decrease of WRN is due to its degradation and ATM/NBS1-dependent phosphorylation of WRN might be important for this to occur. Several lines of evidence indicate a relationship between ATM/ATR-dependent phosphorylation of substrate proteins and degradation of the latter. Hdmx, human homologue of Mdm2, is phosphorylated by ATM following DNA damage, and this phosphorylation is responsible for its poly-ubiquitination and degradation in response to DNA damage (Pereg et al., 2005). Further, the E3 Ubiquitin 
Ligase COP1 is degraded in an ATM-dependent manner after IR and this degradation is mediated by its ATM-dependent phosphorylation and poly-ubiquitination (Dornan et al., 2006). Furthermore, both phosphorylation and degradation of hEXO1 in response to replication fork stalling is controlled by ATR, and mutation of hEXO1 at its phosphorylated sites abrogates ATR-dependent degradation (El-Shemerly et al., 2008). These facts suggest that the degradation of WRN could be also mediated by its ATM/ATR-dependent phosphorylation.

Thus far, it was known that WRN protein interacts with PCNA and DNA polymerase $\delta$ (Cheng et al., 2007). Electron microscopy revealed that WRN binds to DNA templates containing replication forks (Compton et al., 2008), suggesting that WRN participates in the regulation of DNA replication and may stabilize stalled forks. However, it was recently reported that WRN stimulates the extension activity of TLS

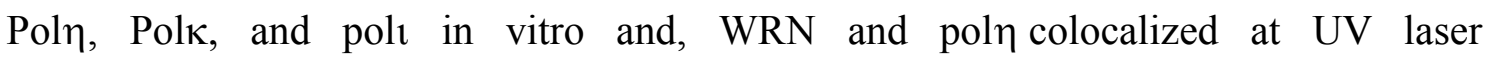
micro-irradiated sites (Kamath-Loeb et al., 2007). These findings suggest that WRN has a positive role in TLS. However, Fig. 3 shows that Werner cells exhibit increased focus formation of Rad18 and Poln, indicating active TLS, without DNA-damaging treatment. Furthermore, Fig. 4 also suggested that WRN represses spontaneous mono-ubiquitination of PCNA, which regulates the switching of DNA polymerase to TLS pols, through its interaction with PCNA and might play a repressive role to TLS pathway, which could lead to somatic mutations. Namely, WRN might contribute to repression of somatic mutations through regulation of switching to TLS pols and prevention of mismatched base pair accumulation. On the other hand, it was reported 
that deficiency of Msh2 or Msh6, which could lead to accumulation of mismatched base pairs, showed chromosome aberration containing breaks, and that both deficiency of mismatch repair and HR repair caused chromatid breaks (Campbell et al., 2006; Vernole et al., 2009). As WRN has also been reported to play a possible role in HR pathway, the combination of mismatched base pair accumulation through un-regulated TLS and abnormal HR regulation may lead to reported genomic deletions in WS cells (Chen et al, 2003; Saintigny et al., 2002). Moreover, the functional interaction of WRN and Rad18/PCNA, shown in Fig. 4, is supported by a report indicating that the sensitivities of WRN(-/-)/RAD18(-/-) double-knockout DT-40 cells to both 4NQO and MMS was almost the same as those of RAD18(-/-) cells (Dong et al., 2007). However, Cheng et al. recently reported that WRN is required for ATM activation and S phase checkpoint in response to interstrand cross-link-induced DNA damage (Cheng et al., 2008), suggesting the role of WRN in S phase-dependent cell cycle checkpoint. Therefore, it might be very important to investigate the detailed role of WRN in both $\mathrm{S}$ phase-checkpoint and translesion synthesis and the possible ramifications of these roles in preventing tumorigenesis.

\section{ACKNOWLEDGMENTS}

The authors would like to thank K. Sperling for NBS cells, Y. Mitsui for human primary fibroblasts, F. Hanaoka for GFP-poln-expressing HeLa cells and P. Jeggo for hTERT-introduced human primary fibroblasts. This work was supported by grants from 
the Ministry of Education, Culture, Sports, Science and Technology of Japan (21310035; JK, 18101002; KK), grants NIH RO1 and CA50519 (DJC), grants from NASA (NNA05CS97G and NNX10AE08G) to SB, a grant from the Cancer Prevention and Research Institute of Texas (RP100644) to SB, and in part by NIFS Collaborative Research Program (NIFS10KOBS022; JK).

\section{CONFLICTS OF INTEREST}

The authors declare that they have no competing financial interests.

\section{Figure Legends}

Figure 1. NBS1-dependent WRN focus formation in response to DNA damage. (A) Formation of WRN foci in response to several DNA-damaging agents. HeLa cells were treated by several DNA-damaging agents as indicated. After 4 hours, cells were fixed and immuno-staining was performed using anti-WRN antibody. (B) Formation of WRN foci in NBS cells after DNA damage. Normal (NBS1-complemented) and NBS cells were treated by IR ( 5 Gy of $\gamma$-ray). After 1 hour, their cells were fixed and immuno-staining was performed using anti-WRN antibody. (C) Physical interaction of WRN with NBS1. Extracts from normal and NBS lymphoblastoid cells, harvested at 30 minutes after 10 Gy of IR, were immunoprecipitated with anti-WRN antibody and the immuno-complexes was detected by Western blot analysis. (D) Focus formation of 
several DNA damage response factors in WS cells. WS cells were treated by IR (5 Gy of $\gamma$-ray). After 30 minutes, their cells were fixed and immuno-staining was performed.

Figure 2. NBS1 interacts with WRN and contributes to ATM-dependent WRN phosphorylation. (A) ATM/NBS1-dependent phosphorylation of WRN. Normal (MRC5), NBS and AT fibroblast cells were irradiated with 5 Gy of $\gamma$-rays. These cells were harvested at the indicated times after IR and analyzed by Western blot using anti-WRN antibody. Phosphorylated WRN was detected as the slower-migrating band. (B) Schematics showing protein structure and design constructs of NBS1. MBD means hMre11-binding domain and AIM means ATM-interacting motif. NBS1-R2 mutant truncates C-terminus (670aa-754aa), FHA-2D mutant substitutes both Gly27 and Arg28 to Asp and BRCT-2D mutant substitutes both Lys150 and Val 151 to Asp. These mutated NBS1 were generated and used in (Sakamoto et al., 2007; Tauchi et al., 2001). (C) Interaction of NBS1 with WRN through the FHA domain. NBS cells, expressing each mutated NBS1 construct, was harvested at 30 minutes after 10 Gy of IR and then were immunoprecipitated with anti-NBS1 antibody and the immuno-complexes was detected by Western blot analysis. (D) WRN phsophorylation was investigated in the NBS cells, expressing FHA-truncating NBS1 or wild type NBS1 (Full) by Western blot analysis. (E) Formation of WRN foci in ATM-deficient cells. AT or HeLa cells were treated by CPT $(1 \mu \mathrm{M})$. After 1 hour, their cells were fixed and immuno-staining was performed using anti-WRN antibody. +caffeine: pre-treatment 
by $1 \mathrm{mM}$ caffeine.

Figure 3. WRN responds to $\mathrm{S}$ phase-dependent DNA damage. (A) IR-induced activation of ATM-related pathway. WS and normal cells were irradiated by 5 Gy of $\gamma$-ray. These cells were harvested at 0.5 hour after IR and analyzed by Western blot using indicated antibodies. (B) S phase-dependent WRN focus formation. Normal primary fibroblasts (TIG-3) were incubated in low serum media for 48 hours and then stimulated to proliferate by exchanging with high serum media (Fukami et al., 1995). After then, these cells were treated by etoposide $(30 \mu \mathrm{M})$ for 4 hours and immuno-staining was performed using anti-WRN and anti-phospho-DNA-PKcs (T2609) antibodies. G1: at 2 hours after serum stimulation. S: at 12 hours after serum stimulation. (C) Formation of poln foci in WRN-knockdown cells. GFP-poln-expressing HeLa cells were transfected by WRN siRNA. After 2 days, their cells were treated by UV $\left(20 \mathrm{~J} / \mathrm{m}^{2}\right)$. After 4 hour, their cells were fixed and the focus formation of GFP-pol $\eta$-was observed and counted under a fluorescent microscope. (D) Formation of Rad18 foci in WS cells after CPT treatment. Normal (48BR) and WS cells were treated by CPT $(2 \mu \mathrm{M})$. After 4 hour, their cells were fixed and immuno-staining was performed using anti-Rad18 antibody and Rad18 foci-positive cells were counted under a fluorescent microscope. (E) supF mutant frequency in WS cells. UV-irradiated or un-irradiated pSP189 was transfected into normal (48BR) and WS cells. After 48 hours, replicated pSP189 was recovered from cells, and supF mutant frequencies were determined as described in Material and Methods. 
Figure 4. WRN interacts with PCNA and might contribute to translesion synthesis. (A)(B) Physical interaction of WRN with PCNA. The extracts from normal or NBS lymphoblastoid cells, harvested at indicated times after 10 Gy of $\gamma$-ray (A) or at $1 \mathrm{~h}$ after $1 \mu \mathrm{M}$ of CPT treatment (B), were immunoprecipitated with anti-WRN antibody or normal rabbit $\mathrm{IgG}$, and then the immuno-complexes was detected by Western blot analysis. Ratios about immunoprecipitated PCNA were calculated to each un-treated sample by ImageJ software. (C) DNA damage-dependent interaction between PCNA and Rad18. The extracts from normal (48BR) or WS cells, harvested at 1 hour after 1 $\mu \mathrm{M}$ of CPT treatment, were immunoprecipitated with anti-Rad18 antibody, and then the immuno-complexes was detected by Western blot analysis. Ratios of immunoprecipitated PCNA were calculated to un-irradiated sample (normal cells) by ImageJ software. (D) Spontaneous ubiquitination of PCNA in WS cells. Normal (48BR) and WS cells were treated by 5 Gy of $\gamma$-ray or $1 \mu \mathrm{M}$ of CPT. These cells were harvested at the indicated times after DNA damage and analyzed by Western blot using anti-PCNA antibody. Ubiquitinated PCNA was detected as the delayed band (Ub). Ratios of ubiquitinated PCNA were calculated to un-irradiated sample (normal cells) by ImageJ software. (E)(F) DNA damage-dependent degradation of WRN. Normal, AT, and NBS lymphoblastoid cells (E) or 48BR cells (F) were treated by $1 \mu \mathrm{M}$ of CPT. These cells were harvested at the indicated times after CPT treatment and analyzed by Western blot using anti-WRN antibody. Ratios were calculated to each un-irradiated sample by ImageJ software. Arrow: WRN protein, *: non-specific bands. ATM 
inhibitor: pre-treatment of KU-55933 [10 $\mu \mathrm{M}, 1$ hour] (Hickson et al., 2004).

Figure 5 WRN participates in the regulation of PCNA mono-ubiquitination and subsequent TLS through interaction with PCNA. WRN is phosphorylated by ATM/NBS1 in response to DNA damage and this phosphorylation leads to a degradation of WRN. PCNA is released from WRN complex following WRN degradation and then could be mono-ubiquitinated by Rad18/Rad6. Mono-ubiquitination of PCNA activates translesion DNA synthesis.

\section{References}

Bartkova, J., Horejsí, Z., Koed, K., Krämer, A., Tort, F., Zieger, K., Guldberg, P., Sehested, M., Nesland, J.M., Lukas, C., Ørntoft, T., Lukas, J., Bartek, J., 2005. DNA damage response as a candidate anti-cancer barrier in early human tumorigenesis. Nature 434, 864-870.

Brosh, R.M. Jr, von Kobbe, C., Sommers, J.A., Karmakar, P., Opresko, P.L., Piotrowski, J., Dianova, I., Dianov, G.L., Bohr, V.A., 2001. Werner syndrome protein interacts with human flap endonuclease 1 and stimulates its cleavage activity. EMBO J. $20,5791-5801$.

Campbell, M.R., Wang, Y., Andrew, S.E., Liu, Y., 2006. Msh2 deficiency leads to chromosomal abnormalities, centrosome amplification, and telomere capping defect. Oncogene 25, 2531-2536.

Casali, P., Pal, Z., Xu, Z., Zan, H., 2006. DNA repair in antibody somatic 
hypermutation. Trends Immunol. 27, 313-321.

Chan, D.W., Chen, B., Prithivirajsingh, S., Kurimasa, A., Story, M.D., Qin, J., Chen, D.J., 2002. Autophosphorylation of the DNA-dependent protein kinase catalytic subunit is required for rejoining of DNA double-strand breaks. Genes Dev. 16, $2333-2338$.

Chen, B.P., Chan, D., Kobayashi, J., Burma, S., Asaithamby, A., Morotomi-Yano, K., Botvinick, E., Qin, J., Chen, D.J., 2005. Cell cycle dependence of DNA-dependent protein kinase phosphorylation in response to DNA double strand breaks. J. Biol. Chem. 280, 14709-14715.

Chen L., Huang S., Lee L., Davalos A., Schiestl R.H., Campisi J., Oshima J., 2003. WRN, the protein deficient in Werner syndrome, plays a critical structural role in optimizing DNA repair. Aging Cell. 2, 191-199.

Cheng, W.H., von Kobbe, C., Opresko, P.L., Arthur, L.M., Komatsu, K., Seidman, M.M., Carney, J.P., Bohr, V.A., 2004. Linkage between Werner syndrome protein and the Mre11 complex via Nbs1. J. Biol, Chem. 279, 21169-21176.

Cheng, W.H., Sakamoto, S., Fox, J.T., Komatsu, K., Carney, J., Bohr, V.A., 2005. Werner syndrome protein associates with gamma H2AX in a manner that depends upon Nbs1. FEBS Lett. 579, 1350-1356.

Cheng, W.H., Muftuoglu, M., Bohr, V.A., 2007. Werner syndrome protein: functions in the response to DNA damage and replication stress in S-phase. Exp. Gerontol. 42, 871-878.

Cheng, W.H., Muftic, D., Muftuoglu, M., Dawut, L., Morris, C., Helleday, T., 
Shiloh, Y., Bohr, V.A., 2008. WRN is required for ATM activation and the S-phase checkpoint in response to interstrand cross-link-induced DNA double-strand breaks. Mol. Biol. Cell. 19, 3923-3933.

Compton, S.A., Tolun, G., Kamath-Loeb, A.S., Loeb, L.A., Griffith, J.D., 2008. The Werner syndrome protein binds replication fork and holliday junction DNAs as an oligomer. J. Biol. Chem. 283, 24478-24483.

Constantinou, A., Tarsounas, M., Karow, J.K., Brosh, R.M., Bohr, V.A., Hickson, I.D., West, S.C., 2000. Werner's syndrome protein (WRN) migrates Holliday junctions and co-localizes with RPA upon replication arrest. EMBO Rep. 1, 80-84.

Dong, Y.P., Seki, M., Yoshimura, A., Inoue, E., Furukawa, S., Tada, S., Enomoto, T., 2007. WRN functions in a RAD18-dependent damage avoidance pathway. Biol. Pharm. Bull. 30, 1080-1083.

Dornan, D., Shimizu, H., Mah, A., Dudhela, T., Eby, M., O'rourke, K., Seshagiri, S., Dixit, V.M., 2006. ATM engages autodegradation of the E3 ubiquitin ligase COP1 after DNA damage. Science 313, 1122-1126.

El-Shemerly, M., Hess, D., Pyakurel, A.K., Moselhy, S., Ferrari, S., 2008. ATR-dependent pathways control hEXO1 stability in response to stalled forks. Nucleic Acids Res. 36, 511-519.

Falck J., Coates J., Jackson S.P., 2005. Conserved modes of recruitment of ATM, ATR and DNA-PKcs to sites of DNA damage. Nature 434, 605-611.

Fukami, J., Tsuji, K., Ueno, A., Ide, T., 1995. Transforming growth factor-beta 1 has both promoting and inhibiting effects on induction of DNA synthesis in human 
fibroblasts. Exp. Cell Res. 216, 107-112.

Gray, M.D., Shen, J., Kamath-Loeb, A.S., Blank, A., Sopher, B.L., Martin, G. M., Oshima, J., Loeb, L.A., 1997. The Werner syndrome protein is a DNA helicase. Nat. Genet. 17, 100-103.

Hickson, I., Zhao, Y., Richardson, C.J., Green, S.J., Martin, N.M., Orr, A.I., Reaper, P.M., Jackson, S.P., Curtin, N.J., Smith, G.C., 2004. Identification and characterization of a novel and specific inhibitor of the ataxia-telangiectasia mutated kinase ATM. Cancer Res. 64, 9152-9159.

Hoege, C., Pfander, B., Moldovan, G.L., Pyrowolakis, G., Jentsch, S., 2002. RAD6-dependent DNA repair is linked to modification of PCNA by ubiquitin and SUMO. Nature 419, 135-141.

Iijima, K., Ohara, M., Seki, R., Tauchi, H., 2008. Dancing on Damaged Chromatin: Functions of ATM and the RAD50/MRE11/NBS1 Complex in Cellular Responses to DNA Damage. J Radiat. Res. 49, 451-464.

Kamath-Loeb, A.S., Lan, L., Nakajima, S., Yasui, A., Loeb, L.A., 2007. Werner syndrome protein interacts functionally with translesion DNA polymerases. Proc. Natl. Acad. Sci. U S A 104, 10394-10399.

Kobayashi, J., Tauchi, H., Sakamoto, S., Nakamura, A., Morishima, K., Matsuura, S., Kobayashi, T., Tamai, K., Tanimoto, K., Komatsu, K., 2002. NBS1 localizes to gamma-H2AX foci through interaction with the FHA/BRCT domain. Curr. Biol. 12, 1846-1851.

Kobayashi, J., Antocia, A., Tauchi, H., Matsuura, S., Komatsu, K., 2004. 
NBS1 and its functional role in the DNA damage response. DNA Repair 3, 855-861.

Lehmann, A.R., 2006. New functions for Y family polymerases. Mol. Cell 24, 493-495.

Masutani, C., Kusumoto, R., Iwai, S., Hanaoka, F., 2000. Mechanisms of accurate translesion synthesis by human DNA polymerase eta. EMBO J. 19, 3100-3109.

Matsuoka, S., Ballif, B., Smogorzewska, A., McDonald, E.R. 3rd, Hurov, K.E., Luo, J., Bakalarski, C.E., Zhao, Z., Solimini, N., Lerenthal, Y., Shiloh, Y., Gygi, S.P., Elledge, S.J., 2007. ATM and ATR substrate analysis reveals extensive protein networks responsive to DNA damage. Science 316, 1160-1166.

Otterlei, M., Bruheim, P., Ahn, B., Bussen, W., Karmakar, P., Baynton, K., Bohr, V.A., 2006. Werner syndrome protein participates in a complex with RAD51, RAD54, RAD54B and ATR in response to ICL-induced replication arrest. J. Cell Sci. $119,5137-5146$.

Parris, C.N., Seidman, M.M., 1992. A signature element distinguishes sibling and independent mutations in a shuttle vector plasmid. Gene 117, 1-5.

Pereg, Y., Shkedy, D., de Graaf, P., Meulmeester, E., Edelson-Averbukh, M., Salek, M., Biton, S., Teunisse, A.F., Lehmann, W.D., Jochemsen, A.G., Shiloh, Y., 2005. Phosphorylation of Hdmx mediates its Hdm2- and ATM-dependent degradation in response to DNA damage. Proc. Natl. Acad. Sci. U S A 102, 5056-5061.

Perry, J.J., Yannone. S., Holden, L.G., Hitomi, C., Asaithamby, A., Han, S., Cooper, P.K., Chen, D.J., Tainer, J.A., 2006. WRN exonuclease structure and molecular mechanism imply an editing role in DNA end processing. Nat. Struct. Mol. Biol. 13, 
Pichierri, P., Rosselli, F, Franchitto, A., 2003. Werner's syndrome protein is phosphorylated in an ATR/ATM-dependent manner following replication arrest and DNA damage induced during the S phase of the cell cycle. Oncogene 22, 1491-1500.

Poot, M., Gollahon, K., Rabinovitch, P.S., 1999. Werner syndrome lymphoblastoid cells are sensitive to camptothecin-induced apoptosis in S-phase. Hum. Genet. 104, 10-14.

Poot, M., Gollahon, K., Emond, M.J,, Silber, J.R., Rabinovitch, P.S., 2002. Werner syndrome diploid fibroblasts are sensitive to 4-nitroquinoline-N-oxide and 8-methoxypsoralen: implications for the disease phenotype. FASEB J. 16, 757-758.

Rass, E., Grabarz, A., Plo, I., Gautier, J., Bertrand, P., Lopez, B.S., 2009. Role of Mre11 in chromosomal nonhomologous end joining in mammalian cells. Nat. Struct. Mol. Biol. 16, 819-824.

Sakamoto, S., Nishikawa, K., Heo, S.J., Goto, M., Furuichi, Y., Shimamoto, A., 2001. Werner helicase relocates into nuclear foci in response to DNA damaging agents and co-localizes with RPA and Rad51. Genes Cells 6, 421-430.

Sakamoto, S., Iijima, K., Mochizuki, D., Nakamura, K., Teshigawara, K., Kobayashi, J., Matsuura, S., Tauchi, H., Komatsu, K., 2007. Homologous recombination repair is regulated by domains at the $\mathrm{N}$ - and C-terminus of NBS1 and is dissociated with ATM functions. Oncogene 26, 6002-6009.

Saintigny, Y., Makienko, K., Swanson, C., Emond, M.J,. Monnat, R.J. Jr, 2002 Homologous recombination resolution defect in werner syndrome. Mol Cell Biol. 22, 
6971-6978.

Sekimoto, T., Oda, T., Pozo, F.M., Murakumo, Y., Masutani, C., Hanaoka, F., Yamashita, T., 2010. The molecular chaperone Hsp90 regulates accumulation of DNA polymerase eta at replication stalling sites in UV-irradiated cells. Mol Cell. 37,79-89.

Stelter, P., Ulrich, H., 2003. Control of spontaneous and damage-induced mutagenesis by SUMO and ubiquitin conjugation. Nature, 425, 188-191.

Stiff, T., Reis, C., Alderton, G.K., Woodbine, L., O'Driscoll, M., Jeggo, P.A., 2005. Nbs1 is required for ATR-dependent phosphorylation events. EMBO J. 24, 199-208.

Tauchi, H., Kobayashi, J., Morishima, K., Matsuura, S., Nakamura, A., Shiraishi, T., Ito, E., Masnada, D., Delia, D., Komatsu, K., 2001. The forkhead-associated domain of NBS1 is essential for nuclear focus formation after irradiation but not essential for hRAD50/hMRE11/NBS1 complex DNA repair activity. J. Biol. Chem. 276, 12-15.

Tauchi, H., Kobayashi, J., Morishima, K., van Gent, D.C., Shiraishi, T., Verkaik, N.S., vanHeems, D., Ito, E., Nakamura, A., Sonoda, E., Takata, M., Takeda, S., Matsuura, S., Komatsu, K., 2002. Nbs1 is essential for DNA repair by homologous recombination in higher vertebrate cells. Nature 420, 93-98.

Tissier, A., Kannouche, P., Reck, M.P., Lehmann, A.R., Fuchs, R.P., Cordonnier, A., 2004. Co-localization in replication foci and interaction of human Y-family members, DNA polymerase pol eta and REVl protein. DNA Repair 3, $1503-1514$. 
Vernole, P., Muzi, A., Volpi, A., Dorio, A.S., Terrinoni, A., Shah, G.M., Graziani, G., 2009. Inhibition of homologous recombination by treatment with BVDU (brivudin) or by RAD51 silencing increases chromosomal damage induced by bleomycin in mismatch repair-deficient tumour cells. Mutat Res. 664, 39-47.

von Kobbe, C., Karmakar, P., Dawut, L., Opresko, P., Zeng, X., Brosh, R.M. Jr, Hickson, I.D., Bohr, V.A., 2002. Colocalization, physical, and functional interaction between Werner and Bloom syndrome proteins. J. Biol. Chem. 277, 22035-22044.

Watanabe, K., Tateishi, S, Kawasuji, M., Tsurimoto, T., Inoue, H., Yamaizumi, M., 2004. Rad18 guides poleta to replication stalling sites through physical interaction and PCNA monoubiquitination. EMBO J. 23, 3886-3896.

Xie, A., Kwok, A., Scully, R., 2009. Role of mammalian Mre11 in classical and alternative nonhomologous end joining. Nat Struct Mol Biol. 16, 814-818.

Yannone, S.M., Roy, S., Chan, D.W., Murphy, M.B., Huang, S., Campisi, J., Chen, D.J., 2001. Werner syndrome protein is regulated and phosphorylated by DNA-dependent protein kinase. J. Biol. Chem. 276, 38242-38248.

Yu, C.E., Oshima, J., Wijsman, E.M., Nakura, J., Miki, T., Piussan, C., Matthews, S., Fu, Y.H., Mulligan, J., Martin, G.M., Schellenberg, G.D., 1997. Mutations in the consensus helicase domains of the Werner syndrome gene. Werner's Syndrome Collaborative Group. Am J Hum Genet. 60, 330-341. 

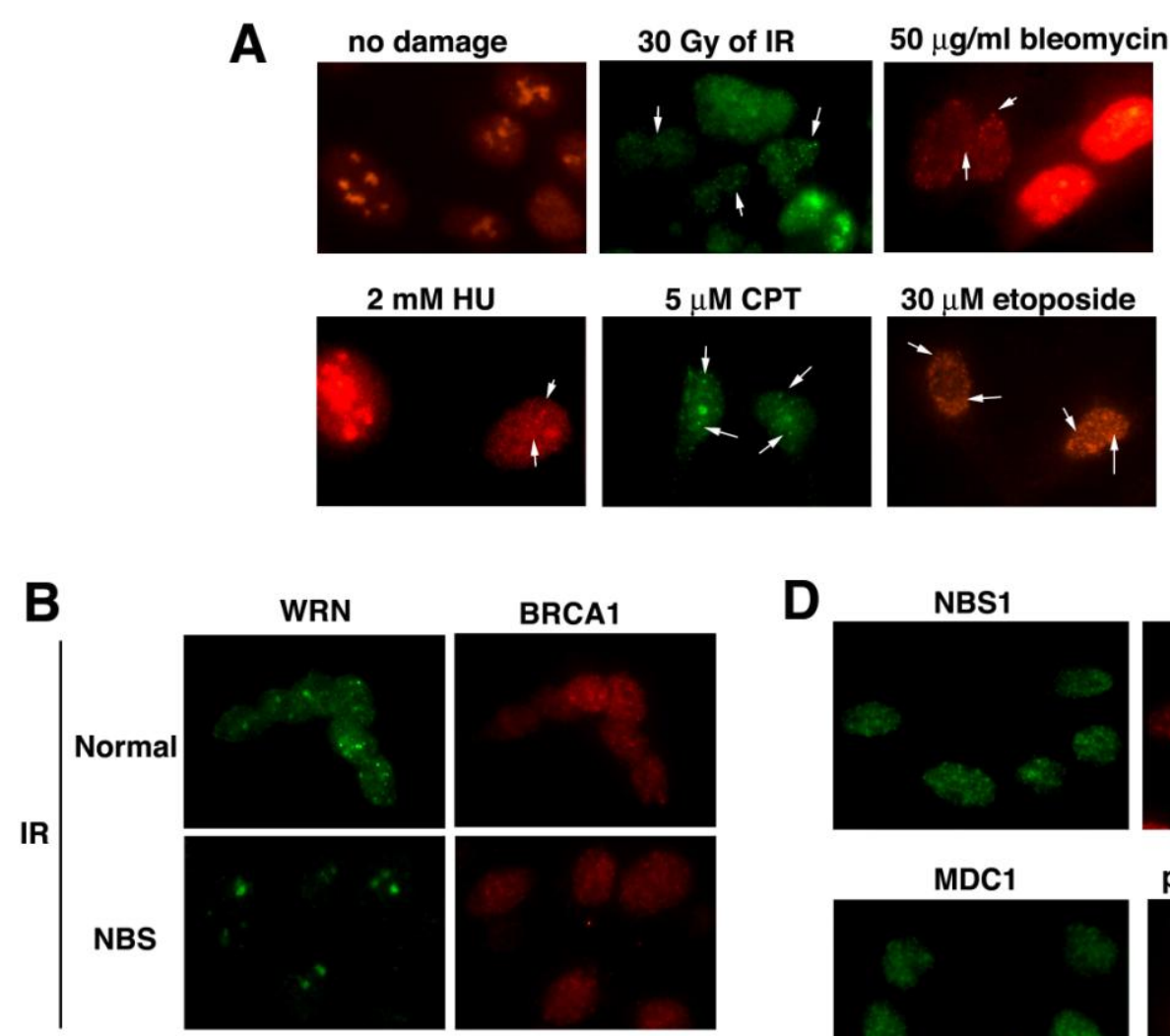

$\gamma-\mathrm{H} 2 \mathrm{AX}$
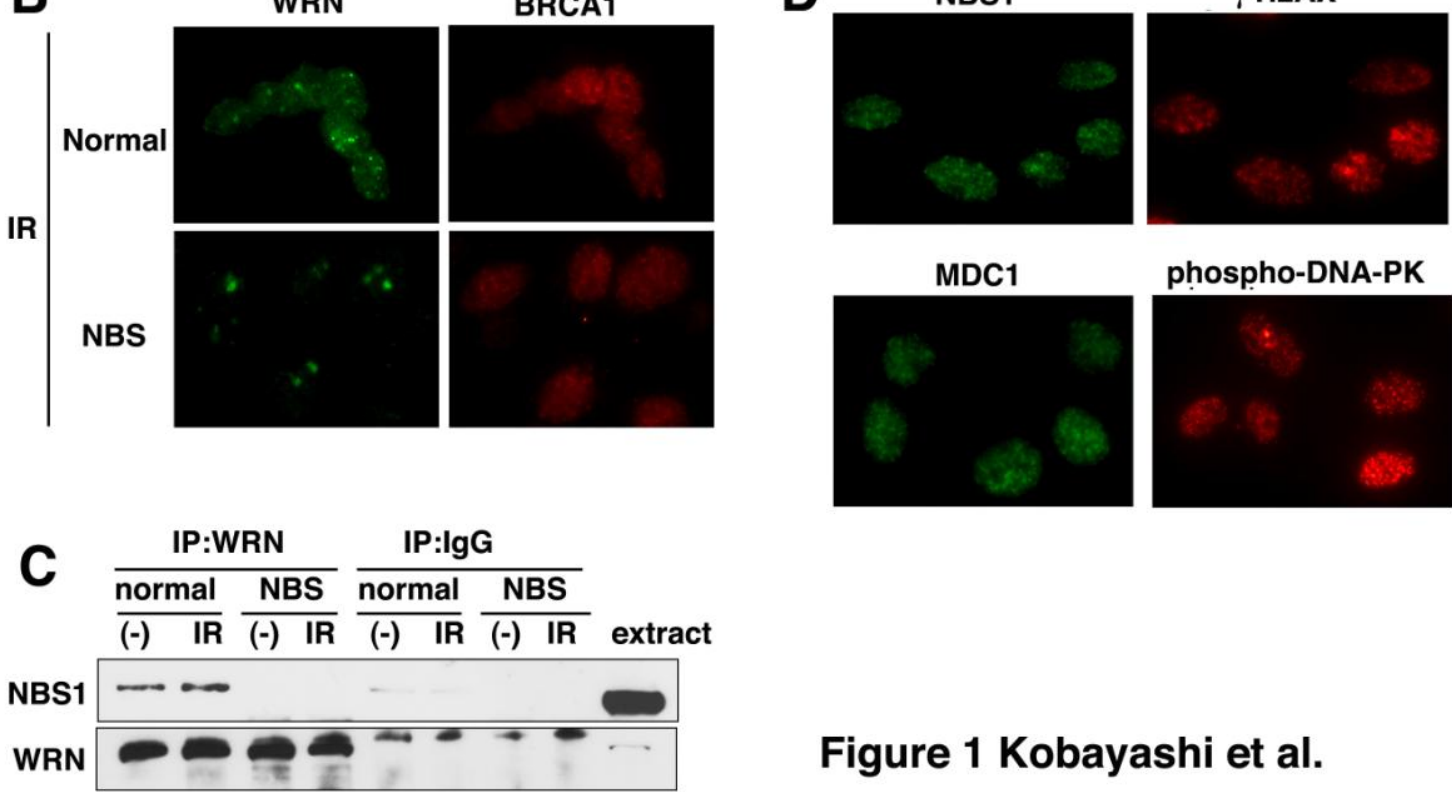

Figure 1 Kobayashi et al. 
A

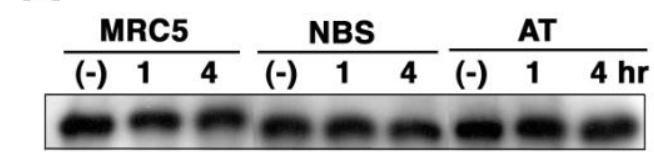

B

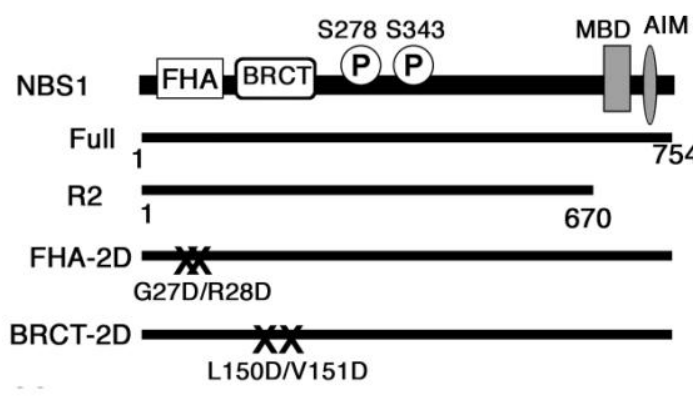

C

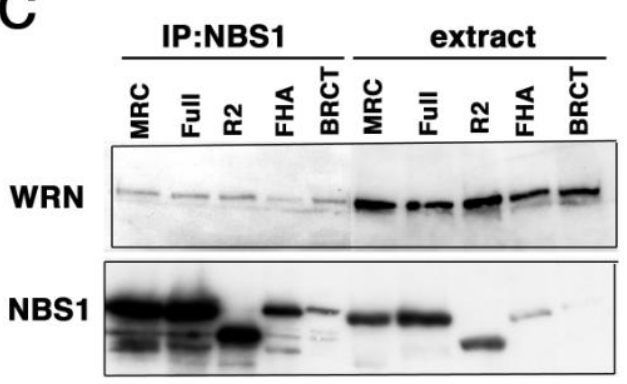

D

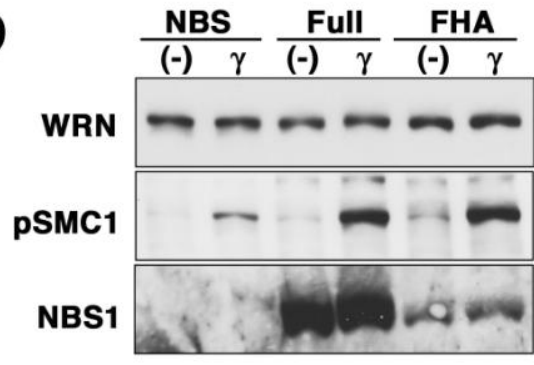

E

WRN

BRCA1

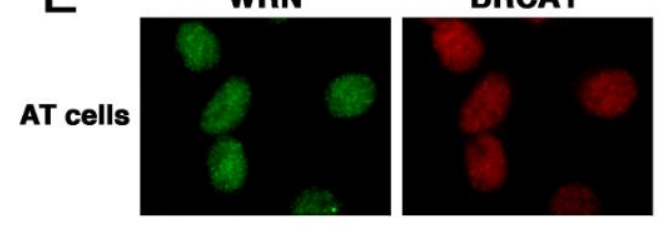

HeLa
CPT
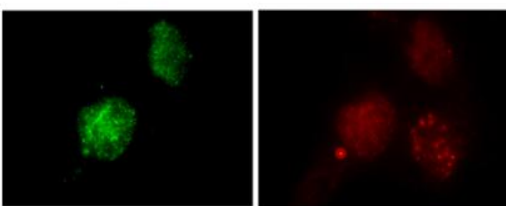

CPT +caffeine
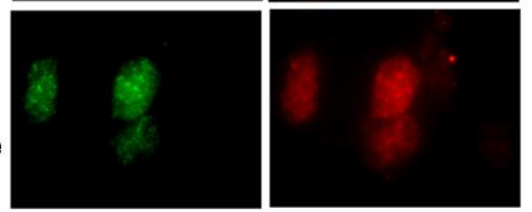

Figure 2 Kobayashi et al. 
A

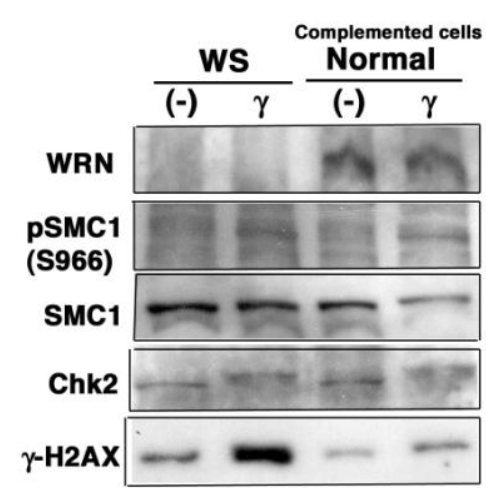

B

B phospho-DNA-PK

WRN

G1

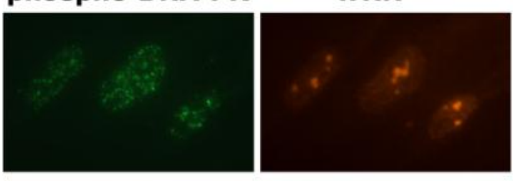

$\mathbf{S}$

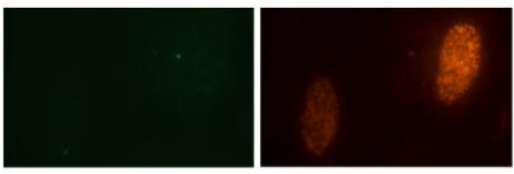

Figure 3 Kobayashi et al.
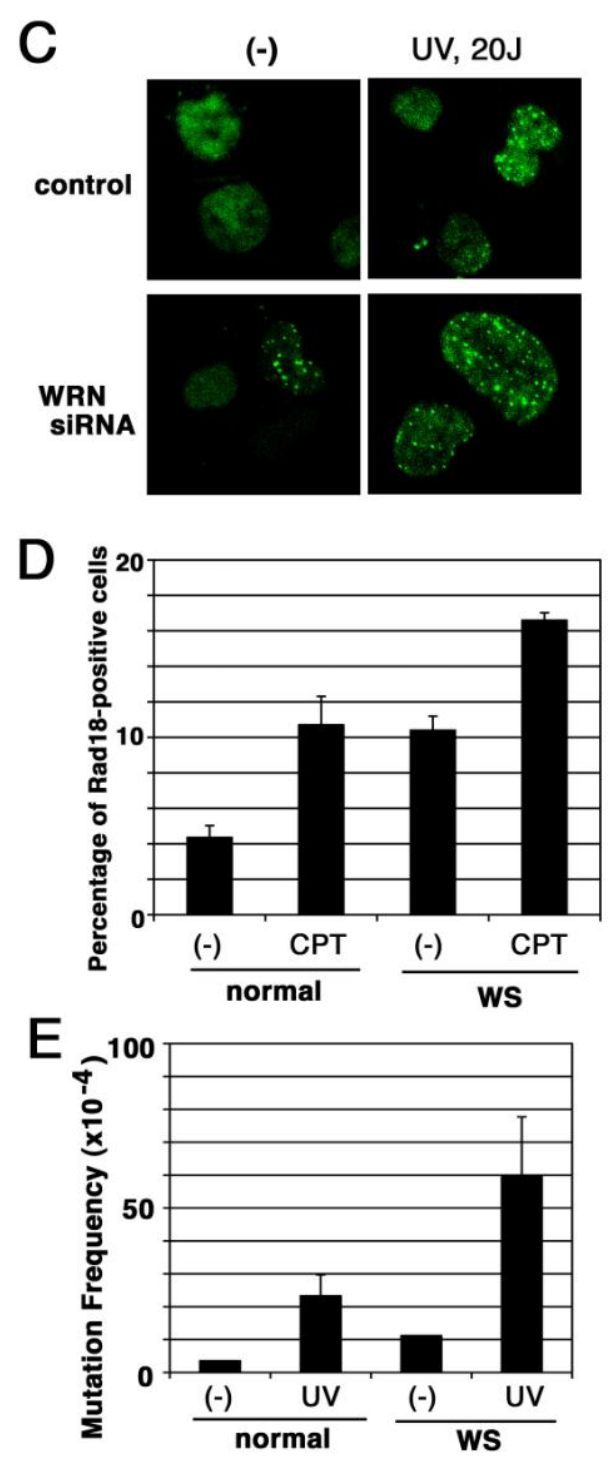

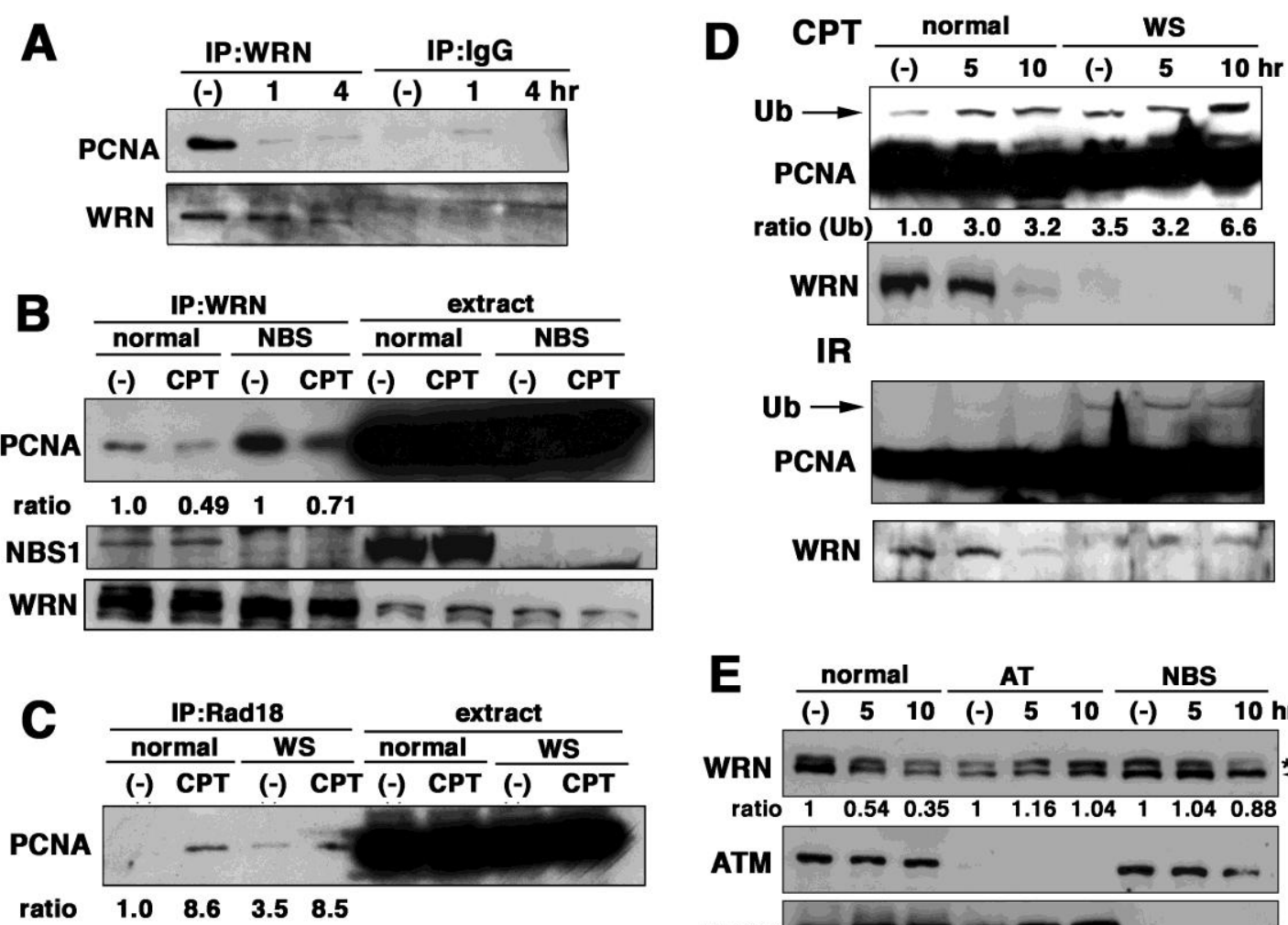

E

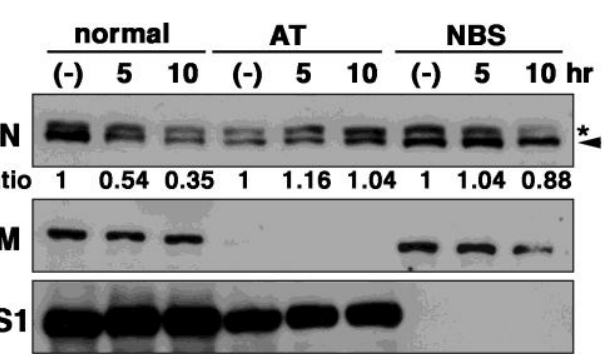

Figure 4 Kobayashi et al.

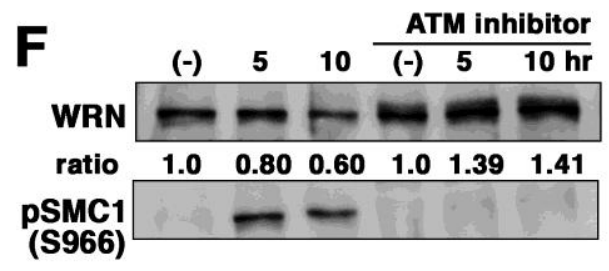

